\title{
Analog front-end measuring biopotential signal with effective offset rejection loop
}

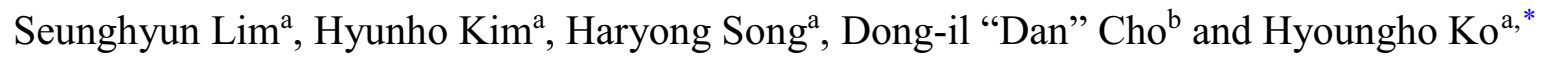 \\ ${ }^{a}$ Department of Electronics, Chungnam National University, Daejeon 305-764, Korea \\ ${ }^{b}$ School of Electrical Engineering, ASRI/ ISRC, Seoul National University, Seoul 151-744, Korea
}

\begin{abstract}
This paper presents an analog front-end (AFE) IC design for recording biopotential signals. The AFE employs a capacitively coupled instrumentation amplifier to achieve a low-noise and high-common mode rejection ratio (CMRR) system. A ripple reduction loop is proposed to reduce the ripple generated by the up-modulating chopper. The low frequency noise is attenuated by an input AC coupling capacitor, and is attenuated again by a DC servo loop. The proposed AFE features a differential gain of $71 \mathrm{~dB}$, and a CMRR of $89 \mathrm{~dB}$, at $50 \mathrm{~Hz}$. Furthermore, the proposed AFE can robustly acquire biopotential signals even in the presence of an input offset and ripples.
\end{abstract}

Keywords: Biopotential analog front-end (AFE), electroencephalograph (EEG), electrocardiogram (ECG), ripple reduction loop, DC servo loop

\section{Introduction}

There has been a growing interest in biopotential acquisition systems for improving the quality of daily life [1]. Because biopotential acquisition devices used in hospitals are bulky and weighty, miniaturized and portable biopotential monitoring systems are required to record signals while causing minimal discomfort. The biopotential signals such as electroencephalogram (EEG) and electrocardiogram (ECG) have small signal amplitudes less than a few $\mathrm{mV}$ in low frequency, thus a low-noise and high CMRR amplifier is required to record high-quality biopotential signals [2]. A capacitively coupled chopper instrumentation amplifier (CCIA) provides efficient topology for these specifications [3]. However, the chopper stabilization employed in the CCIA generates ripple at the instrumentation amplifier (IA) output, due to the up-modulated input offset of the IA [4]. Additionally, the mismatch between the electrodes and the polarization of the $\mathrm{Ag} / \mathrm{AgCl}$ electrodes generates an external offset voltage, which can cause the oversaturation of the IA output.

This paper presents an analog front-end (AFE) IC for recording biopotential signals. The AFE employs the CCIA with multiple feedback loops, which compensate for analog imperfections such as the external offset in the input electrodes and the internal offset in the CCIA; the two feedback loops, a ripple rejection loop (RRL) and a DC servo loop (DSL) are designed to cancel these offsets. In the proposed CCIA, the offset due to the electrode mismatch is inherently attenuated by the AC-coupled

\footnotetext{
* Address for correspondence: Hyoungho Ko, Department of Electronics, Chungnam National University, Daejeon $305-$ 764, Korea. Tel.: +82-42-821-5664; Fax:+82-42-823-5436; E-mail: hhko@cnu.ac.kr.
} 
input stage without DSL. However, DSL is still effective for the reduction of the low-frequency noise and low-frequency signal artifact such as a respiration signal. Thus, an additional DSL is also designed to provide additional poles forthe overall transfer function of the CCIA, and form an additional highpass filter (HPF). As a result, the input offset and low-frequency artifacts are attenuated by a secondorder HPF (CCIA + DSL), in contrast to the first order attenuation used in previous studies [5].

\section{Proposed analog front-end}

The proposed analog front-end (AFE) is shown in Figure 1. The AFE includes the IAs of two stages and fourth order low pass filter (LPF) using a Sallen-Key topology. Each stage of the IA is formed by the CCIA, RRL, and DSL, providing a gain ranging from $23.56 \mathrm{~dB}$ to $35.69 \mathrm{~dB}$. The LPF suppresses the high frequency noise associated with chopping. The cutoff frequency of the LPF is controlled from $100 \mathrm{~Hz}$ to $200 \mathrm{~Hz}$.

Figure 2 shows a simplified schematic of the proposed CCIA. The AC-coupling capacitor blocks the DC offset from the electrodes. The feedback capacitor, $\mathrm{C}_{\mathrm{f}}$, is programmable to adjust the gain of the CCIA. As in Figure 1, the feedback resistor, $\mathrm{R}_{\mathrm{f}}$, should be very large at the appropriate input bias point to achieve linear amplification. In this work, $\mathrm{R}_{\mathrm{f}}$ is implemented by a pseudo-resistor using a well-known MOS-bipolar device to achieve an impedance greater than $100 \mathrm{G} \Omega$. The large impedance of the pseudo-resistor can reduce the output non-linearity due to the non-linear resistance of the pseudo-resistor, as shown in Figure 1. The fully differential amplifier employed in the CCIA is a rail-to-rail, constant $g_{m}$, folded cascode amplifier with a class-A output stage to provide a wide output swing.

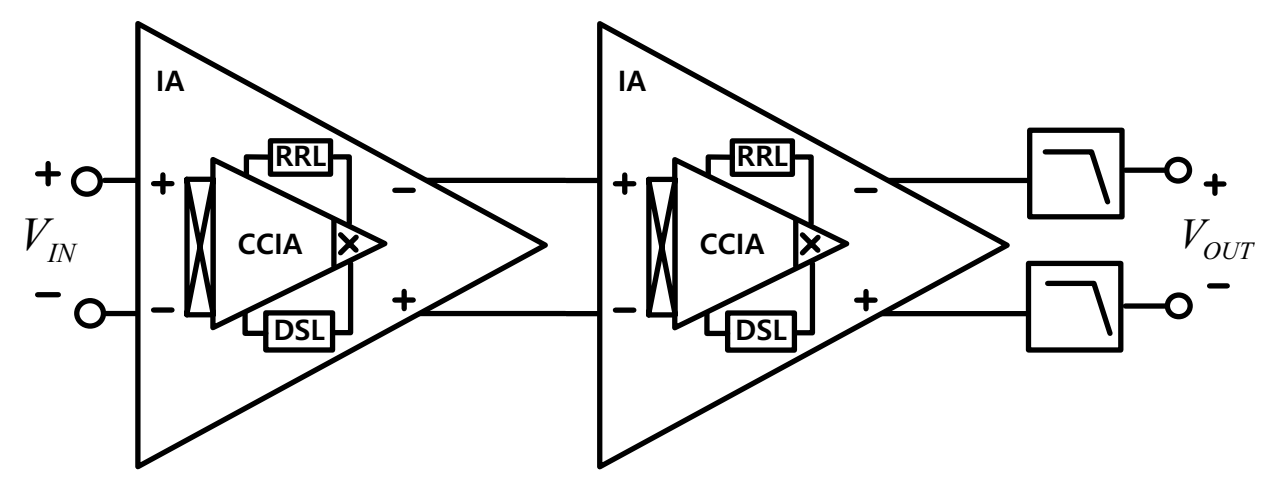

Fig. 1. Proposed analog front-end.

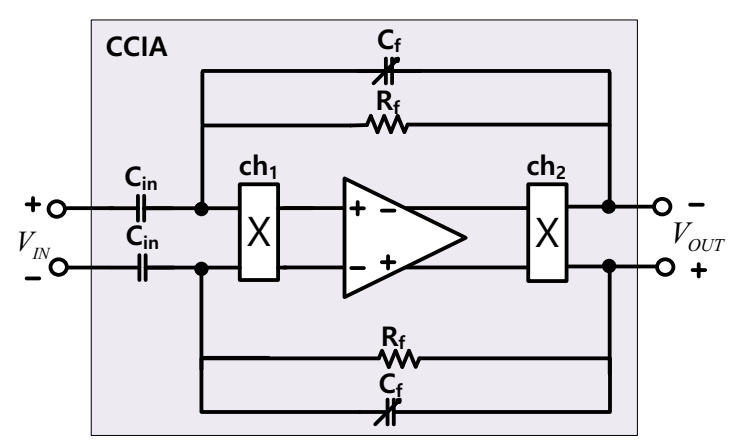

Fig. 2. Schematic of the proposed CCIA. 


$$
\frac{v_{\text {OUT }}}{v_{I N}}=\frac{s C_{i n} R_{f}}{1+s C_{f} R_{f}}=\frac{s C_{i n}}{\frac{1}{R_{f}}+s C_{f}}
$$

Figure 3 shows the fourth order low pass filter (LPF) using a Sallen-Key topology. The LPF removes glitches and the high-frequency noise from the biopotential signal amplified by the IAs. The transfer function of LPF is expressed as:

$$
\frac{v_{\text {OUT }}}{v_{I N}}=\left(\frac{1}{1+s C_{1}\left(R_{1}+R_{2}\right)+s^{2} C_{1} C_{2} R_{1} R_{2}}\right)^{2}
$$

Therefore, the overall transfer function of the AFE is expressed as:

$$
\frac{v_{\text {OUT }}}{v_{I N}}=\left(\frac{s C_{i n} R_{f}}{1+s C_{f} R_{f}}\right)^{2}\left(\frac{1}{1+s C_{1}\left(R_{1}+R_{2}\right)+s^{2} C_{1} C_{2} R_{1} R_{2}}\right)^{2}
$$

\subsection{Ripple reduction loop}

Figure 4 shows a simplified block diagram of the CCIA with the RRL. When an offset voltage, $V_{\text {os, }}$ is present as shown in Figure 4, a ripple is generated at $V_{\text {OUT }}$ by the up-modulating chopper, $\mathrm{ch}_{2}$. This ripple is demodulated to a low frequency by the feedback chopper, $\mathrm{ch}_{3}$, and is filtered by a Miller integrator. $\mathrm{C}_{\mathrm{OL} 1}$ converts the filtered voltage into a current that is fed back to the input of the CCIA. The feedback current is modulated by the chopper, $\mathrm{ch}_{4}$, and is demodulated by the chopper, $\mathrm{ch}_{1}$. Thus, the offset in front of the amplifier, $\mathrm{V}_{\mathrm{OS}}$, is rejected by this negative feedback loop. Consequently, the ripple caused by the offset voltage is reduced. Here, the RRL does not affect the transfer function for the low-frequency range because the RRL operates as a notch filter at the chopper frequency, from the perspective of the demodulated output signal.

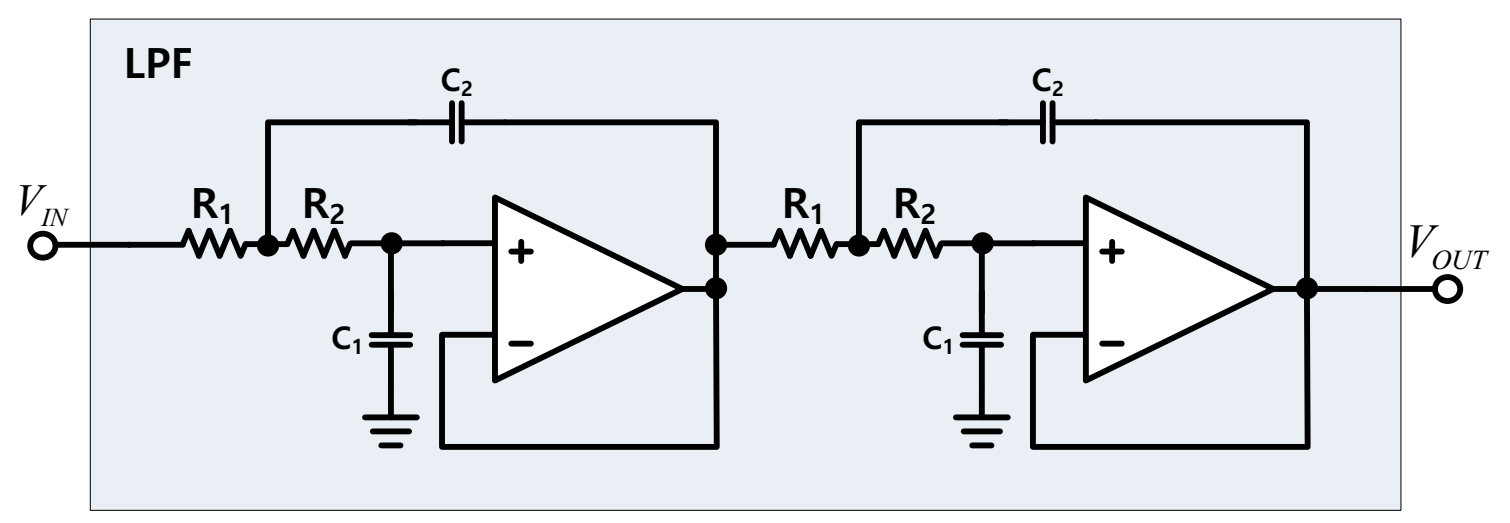

Fig. 3. Fourth order LPF of Sallen-Key topology. 


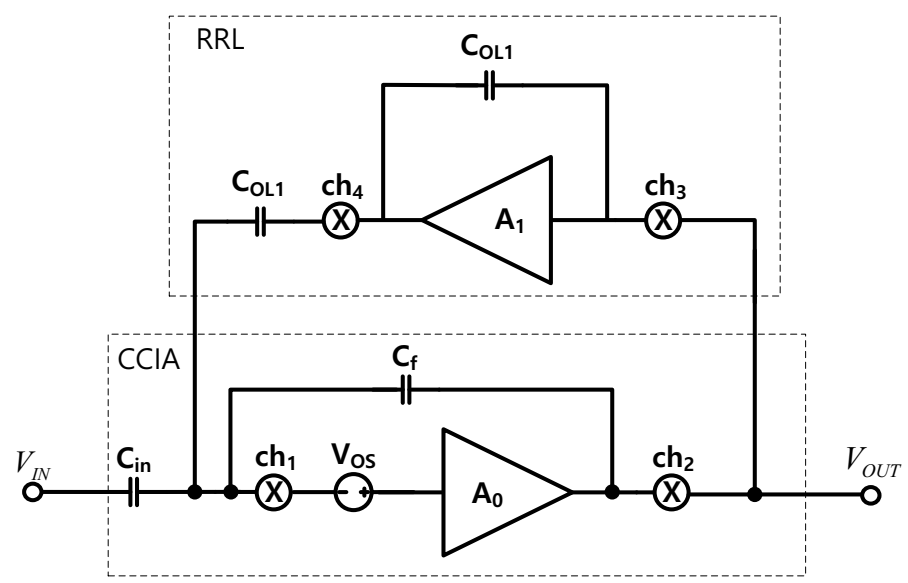

Fig. 4. Simplified block diagram of the RRL and CCIA.

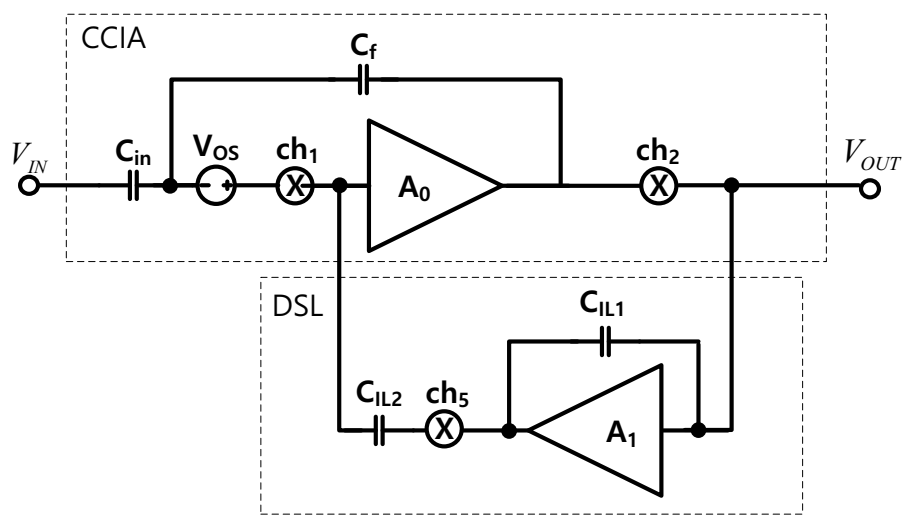

Fig. 5. Simplified block diagram of the CCIA and DSL.

\subsection{DC servo loop}

In the proposed CCIA, the input DC offset is inherently attenuated by the AC-coupling capacitor $\mathrm{C}_{\mathrm{in}}$. However, respiration artifact and other low-frequency noise cannot be rejected completely with the sole HPF characteristics of the CCIA; thus, an additional DSL is designed. Figure 5 shows a simplified block diagram of the CCIA with the DSL. Assuming an offset voltage and low-frequency artifacts, $\mathrm{V}_{\mathrm{OS}}$ is attenuated by the first-order HPF, as shown in Figure 5. However, the remaining low-frequency signal is modulated by $\mathrm{ch}_{1}$, demodulated by $\mathrm{ch}_{2}$, and filtered by the Miller integrator. The output of the Miller integrator is modulated to a high frequency by $\mathrm{ch}_{5}$ and converted into a current that is fed back to the CCIA by $\mathrm{C}_{\mathrm{IL} 2}$. During this negative feedback process, $\mathrm{V}_{\mathrm{OS}}$ is rejected. Here, the DSL operates as an additional HPF in the input stage.

\section{Results and discussions}

Figure 6 illustrates the layout of the proposed circuit, which includes the biopotential AFE and 12bit successive approximation register (SAR) analog-to-digital converter (ADC). When the offset volt- 
age causes a ripple, the output can be saturated. The proposed RRL reduces the ripple to obtain a suitable output signal. Figure 7 shows the effect of the RRL when an offset voltage of $10 \mathrm{mV}$ is present. Over time the ripple decreases, and a sinusoidal wave (without ripple) is observed after 1 second at a steady state. Figure 8 illustrates the effect of the DSL. When the DSL operates, it adds additional poles to the input HPF; thus, the cutoff frequency of the IA adjusts to $3.2 \mathrm{~Hz}$, rejecting the low-frequency noise and respiration artifact. Figure 9 illustrates the differential gain and CMRR of the IA. The $\mathrm{Ag} / \mathrm{AgCl}$ electrode is modeled utilizing a $51 \mathrm{k} \Omega$ resistor and $47 \mathrm{nF}$ capacitor connected in parallel. A CMRR simulation is performed under the worst-case input mismatch condition. The electrode impedance model is connected to only one input IA port. Assuming the modeled electrode is connected to only one of the two IA inputs, the mismatch demonstrates the worst-case scenario. In this situation, a differential gain of $71 \mathrm{~dB}$ and a CMRR of $89 \mathrm{~dB}$ are observed at $50 \mathrm{~Hz}$.

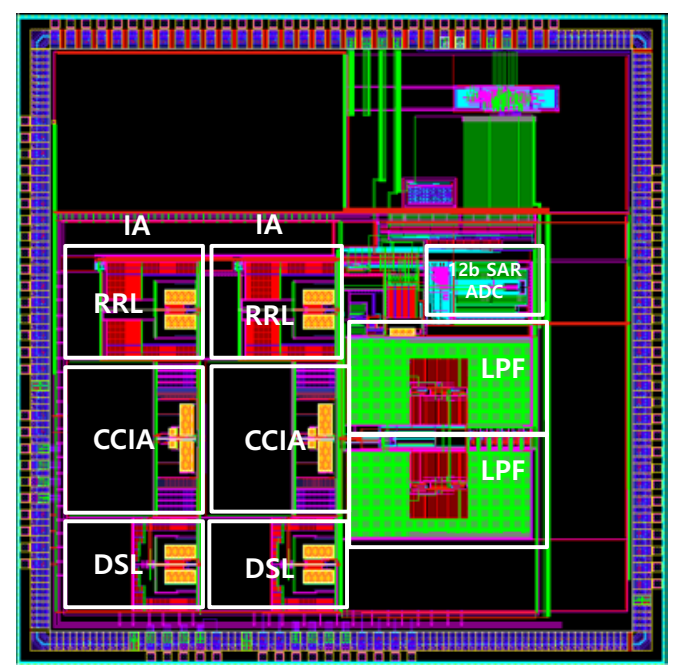

Fig. 6. Layout view.

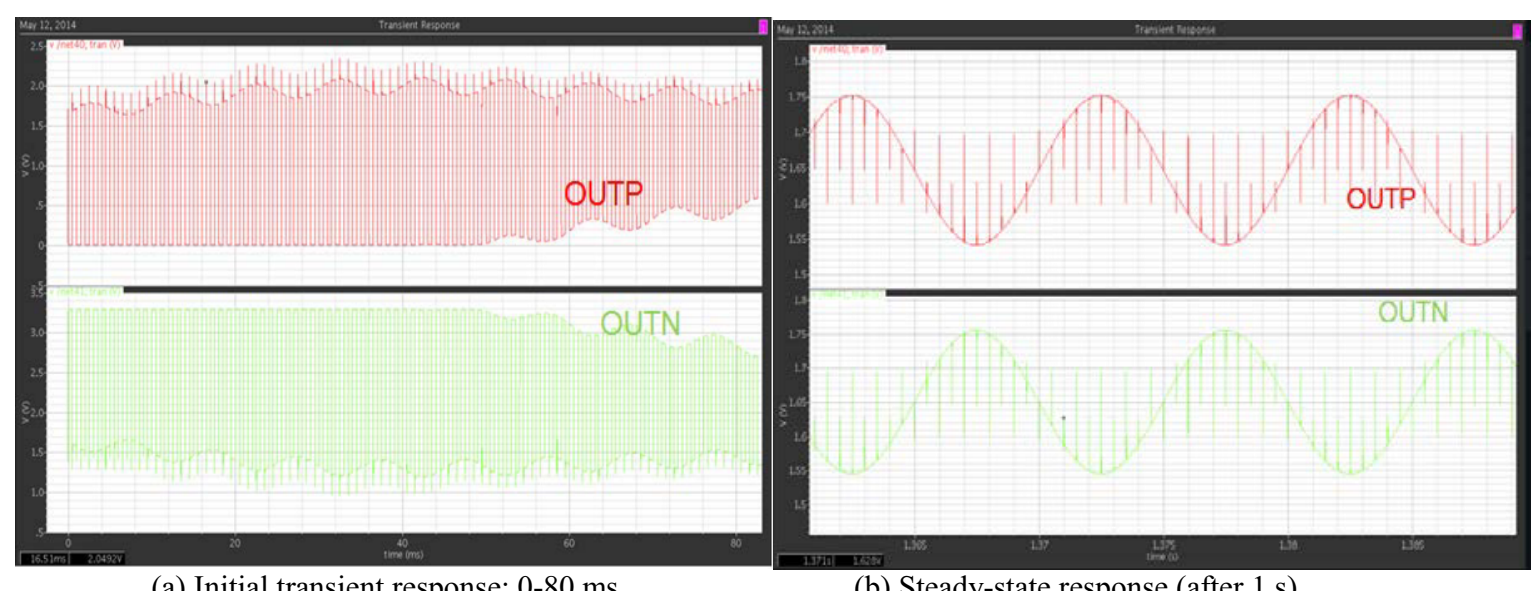

(a) Initial transient response: 0-80 ms.

(b) Steady-state response (after $1 \mathrm{~s}$ ).

Fig. 7. Effect of the RRL. 


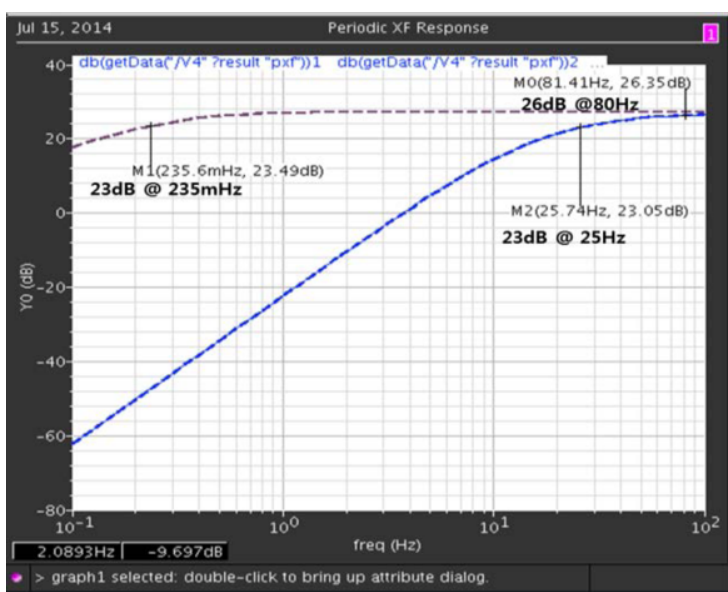

Fig. 8. Effect of the DSL.

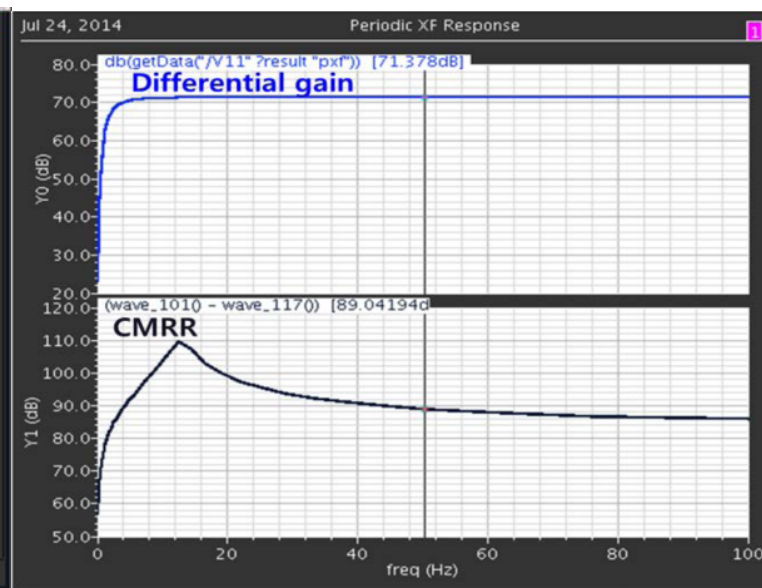

Fig. 9. Differential gain and CMRR of the two stage IAs.

Table 1

Performance Summary

\begin{tabular}{|l|l|l|l|}
\hline & {$[4]$} & {$[5]$} & This work \\
\hline Technology & $0.7 \mu \mathrm{m}$ & $65 \mathrm{~nm}$ & $0.18 \mu \mathrm{m}$ \\
\hline Supply current $(\mu \mathrm{A})$ & 230 & 1.8 & 30 \\
\hline Supply voltage $(\mathrm{V})$ & 5 & 1 & 3.3 \\
\hline AC offset reduction & $\begin{array}{l}\text { First-order reduction with } \\
\text { the Miller integrator }\end{array}$ & $\begin{array}{l}\text { First-order reduction with the } \\
\text { SC integrator }\end{array}$ & $\begin{array}{l}\text { First-order reduction with the } \\
\text { Miller integrator }\end{array}$ \\
\hline DC offset cancelation & Not included & First-order reduction & Second-order reduction \\
\hline CMRR $(\mathrm{dB})$ & 120 & 134 & 89 \\
\hline Input noise $(\mathrm{nV} / \sqrt{\mathrm{Hz}})$ & Not available & 60 & 160 \\
\hline
\end{tabular}

\section{Conclusion}

A biopotential acquisition AFE IC is presented. To reject the offset voltage, low-frequency noise, and respiration artifact, the ripple reduction loop (RRL) and DC servo loop (DSL) are proposed. The results demonstrate that the RRL and DSL effectively reject the ripples and low-frequency noise, respectively. The performance comparisons are given in Table $1[4,5]$. The differential gain and CMRR of the proposed IA are $71 \mathrm{~dB}$ and $89 \mathrm{~dB}$, respectively, at $50 \mathrm{~Hz}$.

\section{Acknowledgments}

This research was supported by the Happy Tech. Program through the National Research Foundation of Korea (NRF), funded by the Ministry of Education, Science and Technology (2010-0020852). This work was also supported by Chungnam National University. This work was also supported by IC Design Education Center (IDEC) and IDEC Platform Center (IPC). 


\section{References}

[1] R.F. Yazicioglu, P. Merken, R. Puers and C. Van Hoof, A $60 \mu \mathrm{W} 60 \mathrm{nV} / \sqrt{\mathrm{Hz}}$ readout front-end for portable biopotential acquisition systems, IEEE Journal of Solid-State Circuits 42.5 (2007), 1100-1110.

[2] W.M. Chen, W.C. Yang, T.Y. Tsai, H. Chiueh and C.Y. Wu, The design of CMOS general-purpose analog front-end circuit with tunable gain and bandwidth for biopotential signal recording systems, 2011 Annual International Conference of the IEEE on Engineering in Medicine and Biology Society, 2011, pp. 4784-4787.

[3] D. Yeager, F. Zhang and A. Zarrasvand, A 9 A, addressable gen2 sensor tag for biosignal acquisition, IEEE Journal of Solid-State Circuits 45.10 (2010), 2198-2209.

[4] R. Wu, K.A. Makinwa and J.H. Huijsing, A chopper current-feedback instrumentation amplifier with a $1 \mathrm{mHz}$ noise corner and an AC-coupled ripple reduction loop, IEEE Journal of Solid-State Circuits 44.12 (2009), 3232-3243.

[5] Q. Fan, F. Sebastiano, J.H. Huijsing and K.A. Makinwa, A $1.8 \mathrm{~W} 60 \mathrm{nV} \mathrm{Hz}$ capacitively-coupled chopper instrumentation amplifier in $65 \mathrm{~nm}$ CMOS for wireless sensor nodes, IEEE Journal of Solid-State Circuits 46.7 (2011), 1534-1543. 